\title{
Mechanism of Amphotericin B Resistance in Leishmania donovani Promastigotes
}

\author{
NICOLAS MBONGO,${ }^{1}$ PHILIPPE M. LOISEAU, ${ }^{2 *}$ MARIE A. BILLION, ${ }^{1}$ AND MALKA ROBERT-GERO ${ }^{1}$ \\ Institut de Chimie des Substances Naturelles, CNRS, F-91190, Gif sur Yvette, ${ }^{1}$ and Biologie et Contrôle \\ des Organismes Parasites, Faculté de Pharmacie, Université de Paris-Sud, F-92296, \\ Châtenay-Malabry, ${ }^{2}$ France
}

Received 23 June 1997/Returned for modification 17 November 1997/Accepted 3 December 1997

\begin{abstract}
Amphotericin B (AmB)-resistant Leishmania donovani promastigotes were selected by increasing drug pressure, and their biological features were compared with those of the wild-type parent strain. The 50\% inhibitory concentration for resistant cells was 20 times higher than that for the wild-type. Resistance was stable after more than $\mathbf{4 0}$ passages in drug-free medium, and resistant promastigotes were infective to macrophages in vitro but lost their virulence in vivo. They had 2.5 times longer generation time, decreased AmB uptake, and increased AmB efflux in comparison to the wild type. Fluorescence measurement with a specific plasma membrane probe, 1-[4-(trimethylammonio)-1,6-diphenylhexa]-1,3,5-triene, showed increased membrane fluidity in drug-resistant promastigotes. Analysis of lipid composition showed that in resistant cells saturated fatty acids were prevalent, with stearic acid as the major fatty acid, and the major sterol was an ergosterol precursor, the cholesta-5, 7, 24-trien-3ß-ol and not ergosterol as in the AmB-sensitive strain.
\end{abstract}

Leishmania donovani, a protozoan parasite, is the causative agent of visceral leishmaniasis, a disease which is fatal in the absence of treatment. Its incidence is about 0.5 million cases annually (28). Pentavalent antimonials (Pentostam and Glucantime) are the first-line drugs, but refractory strains to these drugs are increasingly prevalent in areas of endemicity $(26,33)$. Amphotericin B (AmB) is a polyene antibiotic which binds preferentially to ergosterol, the major sterol of fungi, Leishmania, and Trypanosoma cruzi. This antibiotic is a second-line treatment for leishmaniasis (1), but side effects limit its use. Recently, AmB-lipid formulations (AmBisome and Amphocil) have been developed with reduced toxicity and an improved therapeutic index. They represent an alternative for the treatment of visceral leishmaniasis $(6,23,31)$. As AmB formulations are increasingly being used for the treatment of visceral leishmaniasis and probably mucocutaneous leishmaniasis, we decided to investigate the development and mechanism of resistance. Resistance to $\mathrm{AmB}$ is uncommon in fungi but has been reported previously (16). The aim of our work was to establish a line of AmB-resistant L. donovani promastigotes in vitro and to study the molecular basis of this resistance.

\section{MATERIALS AND METHODS}

Chemical compounds. AmB, cholic acid, bis(trimethylsilyl)trifluoroacetamide (BSTFA), allopurinol riboside, and paromomycin were purchased from Sigma Chemicals (Saint Quentin Fallavier, France). Ketoconazole was purchased from ICN Biomedicals (Orsay, France). Pentamidine isethionate was a gift from Roger Bellon (Neuilly sur Seine, France). Taxotere was a gift from P. Potier (ICSN, Gif sur Yvette, France). Difluoromethylornithine (DFMO) was a gift from Merrel Dow (Strasbourg, France). Sinefungin was purchased from RhônePoulenc Rorer (Vitry sur Seine, France). Boron trifluoride etherate was purchased from Fluka AG (Buchs, Switzerland), and analytical grade (high-performance liquid chromatography [HPLC]) acetonitrile and methanol were obtained from SDS (Peypin, France). Nile red reagent and 1-[4-(trimethylammonio)-1,6diphenylhexa]-1,3,5-triene (TMA-DPH) were purchased from Molecular Probes (Eugene, Oreg.).

* Corresponding author. Mailing address: Biologie et Contrôle des Organismes Parasites, Faculté de Pharmacie, Université de Paris-Sud, F-92296, Châtenay-Malabry, France. Phone: 331468355 54. Fax: 33 1468355 57. E-mail: philippe.loiseau@cep.u-psud.fr.
Isolation of AmB-resistant Leishmania. AmB-resistant clones were obtained from wild-type L. donovani DD8 promastigotes (strain MHOM/IN/80/DD8) by increasing drug concentration in culture medium as previously described for sinefungin (29).

Determination of AmB content of treated promastigotes. (i) Parasite treatment and cell extracts. Promastigotes were cultured in Erlenmeyer flasks at an initial density of $0.5 \times 10^{6}$ to $1 \times 10^{6}$ promastigotes $/ \mathrm{ml}$ in $50 \mathrm{ml}$ of RPMI 1640 medium (Gibco, Eragny, France) with $10 \%$ inactivated fetal calf serum supplemented with $2 \mathrm{mM}$ glutamin and streptomycin $(5 \mu \mathrm{g} / \mathrm{ml})$ and penicillin $(5 \mathrm{IU} / \mathrm{ml})$. Flasks were placed in an orbital incubator (Gallenkamp) under continuous shaking $(150 \mathrm{rpm})$ at $27^{\circ} \mathrm{C}$. When Leishmania cultures reached a density of $20 \times$ $10^{6}$ to $25 \times 10^{6} / \mathrm{ml}$ (logarithmic phase) various concentrations of the drug were added, and cells were incubated for $2 \mathrm{~h}$. Promastigotes were harvested by centrifugation and washed twice with large volumes of cold phosphate-buffered saline (PBS; $\mathrm{pH} 7.5$ ). Pellets were resuspended in $200 \mu \mathrm{l}$ of an aqueous solution of $20 \mathrm{mM}$ cholic acid for $24 \mathrm{~h}$ at $4^{\circ} \mathrm{C}$ and then centrifuged $\left(11,000 \times g, 1 \mathrm{~h}, 4^{\circ} \mathrm{C}\right)$. An equal volume of cold ethanol was added to the supernatant, and the mixture was kept in an ice bath for $1 \mathrm{~h}$ to precipitate the proteins. The samples were then centrifuged $\left(11,000 \times g, 1 \mathrm{~h}, 4^{\circ} \mathrm{C}\right)$, and 150 to $200 \mu \mathrm{l}$ of the clear supernatant was analyzed by HPLC for AmB determination.

HPLC analysis. Reverse-phase chromatography analysis on a Novapack C18 reversed-phase column ( 3.9 by $150 \mathrm{~mm}$.) was performed by previously described methods $(4,18)$ with a liquid chromatograph equipped with a solvent delivery system control (Waters $600 \mathrm{E}$; Millipore), a Waters 717 plus Autosampler with temperature control system $\left(4^{\circ} \mathrm{C}\right.$ for $\mathrm{AmB}$ samples), and a photodiode array detector and reporting integrator (Waters 990). The detection was at $408 \mathrm{~nm}$, corresponding to the maximum absorbance for $\mathrm{AmB}$. The mobile phase was an isocratic gradient of $5 \mathrm{mM}$ EDTA-acetonitrile $(60: 40$, vol/vol) delivered at a flow rate of $1 \mathrm{ml} / \mathrm{min}$. The $5 \mathrm{mM}$ EDTA solution was made in PBS and adjusted to $\mathrm{pH}$ 7.05 with orthophosphoric acid. Standard concentrations of $0.0125,0.125,0.25$, $0.5,1$, and $5 \mu \mathrm{M}$ were made by dissolving $\mathrm{AmB}$ in mobile phase or methanol. Detection limit was found at $0.5 \mathrm{pmol} / \mathrm{mg}$ of protein

Drug efflux. Leishmania promastigotes treated with drug were harvested as mentioned above and transferred to fresh drug-free medium and incubated for various times prior to extraction.

Fluorescent probes. TMA-DPH was prepared as a $2 \mathrm{mM}$ stock solution in dimethylformamide and was stored at $4^{\circ} \mathrm{C}$. An intermediate dilution of $50 \mu \mathrm{M}$ was prepared daily from the $2 \mathrm{mM}$ stock with PBS, agitated vigorously for $10 \mathrm{~min}$ to eliminate the solvent, and used for staining. Nile red was prepared at 100 $\mu \mathrm{g} / \mathrm{ml}(0.3 \mu \mathrm{M})$ in acetone and was conserved at $4^{\circ} \mathrm{C}$ in a dark environment.

Determination of membrane fluidity by flow cytometry. AmB-resistant and wild-type promastigotes were diluted with PBS to a cellular density of $10^{7}$ promastigotes/ml and then stained with $2 \mu \mathrm{M}$ TMA-DPH for 2 min before being analyzed by flow cytometry. Membrane fluidity was assessed by measurement of the fluorescence anisotropy $(r)$ of TMA-DPH following its insertion into the plasma membrane of promastigotes $(9,11)$. The EPICS flow cytometer (Coulter, Hialeah, Fla.) had a $100-\mu \mathrm{m}$-diameter nozzle and standard optics such that each cell was exposed and measured for about $1.5 \mu$ s, i.e., 2 orders of magnitude above the fluorescence lifetime of these probes. The argon laser (Spectra-Physics 2025-

05 ) with vertically polarized output was set at 351 to $364 \mathrm{~nm}$ and $50 \mathrm{~mW}$. The 
signals were processed on an analog card linked to a 1,024-channel analog-todigital converter (ADC) to derive the blue emission anisotropy $(r)$ as previously described (30). The forward-angle light scatter was used to gate the analysis upon intact cells. The mean $r$ value was calculated for 10,000 cells.

Determination of lipids. AmB-resistant and wild-type promastigotes were prepared as described above for fluidity measurements, stained with Nile red (100 $\mathrm{ng} / \mathrm{ml}$ ) for $5 \mathrm{~min}$, and then individually analyzed by flow cytometry. The excitation wavelength of the same argon laser was set at $488 \mathrm{~nm}$ and $400 \mathrm{~mW}$. The fluorescent emission was filtered through a 515-nm long-pass filter (interference and absorbance) and split with a 590-nm dichroic mirror, sending the red component through a 610-nm long-pass filter to one photomultiplier and the yellow component through a 560-nm short-pass filter to another photomultiplier. Cellular fluorescence in the absence of dye was negligible. In each analysis, 10,000 promastigotes were studied. The intensities of red and yellow fluorescence were measured as previously described (21).

Extraction and analysis of free sterols and fatty acids. Promastigotes were grown as described above in three Erlenmeyer flasks in a total volume of 1 liter of culture medium. For cell treatment a volume of drug in $10 \%$ dimethyl sulfoxide in water corresponding to an appropriate final concentration was added, and cultures were incubated at $27^{\circ} \mathrm{C}$ for various times at $150 \mathrm{rpm}$ in an orbital incubator (Gallenkamp). Cells were harvested, washed, and pooled, and the pellet was resuspended in $20 \mathrm{ml}$ of dichloromethane-methanol $(2: 1, \mathrm{vol} / \mathrm{vol})$ for about $24 \mathrm{~h}$ at $4^{\circ} \mathrm{C}$. After centrifugation $\left(11,000 \times g, 1 \mathrm{~h}, 4^{\circ} \mathrm{C}\right)$ the extract was evaporated under vacuum. The residue and the pellet were saponified with $30 \%$ $\mathrm{KOH}$ in methanol at $80^{\circ} \mathrm{C}$ for $2 \mathrm{~h}$. Sterols were extracted with petroleum ether, which was thereafter evaporated, and the residue was dissolved in dichloromethane. An aliquot of clear yellow sterol solution was added to 2 volumes of BSTFA, and the sealed tubes were heated at $80^{\circ} \mathrm{C}$ for $1 \mathrm{~h}$. The trimethylsilyl (TMS) ethers of sterols were subjected to gas chromatography/mass spectrometry (GC/MS) analysis following previously described methods with some modifications $(10,19$, 22).

Aqueous phases of $\mathrm{KOH}$ methanolic extracts were acidified with $6 \mathrm{~N}$ sulfuric acid to $\mathrm{pH} 3$. Fatty acids were extracted with petroleum ether and diethyl ether and transesterified by $\mathrm{BF}_{3}$-methanol at room temperature for $2 \mathrm{~h}$. Methyl esters were extracted with diethyl ether, redissolved in methanol-diethyl ether (1:1) prior to gas-liquid chromatography (GLC)/MS analysis.

GLC was performed with a Varian model 3400 chromatograph equipped with DB5 columns (methyl/phenylsiloxan ratio, 95/5; dimensions, $30 \mathrm{~m}$ by $0.25 \mathrm{~mm}$ ). The gas carrier was $\mathrm{He}(1 \mathrm{ml} / \mathrm{min})$. Analysis conditions were as follows: the column was kept at $270^{\circ} \mathrm{C}$, the injector was kept at $300^{\circ} \mathrm{C}$ (splitless), and the detector was kept at $300^{\circ} \mathrm{C}$ (isothermic conditions for sterols). The linear gradient for methyl esters was from 150 to $180^{\circ} \mathrm{C}$ at $10^{\circ} \mathrm{C} / \mathrm{min}$. MS conditions were $280^{\circ} \mathrm{C}, 70 \mathrm{eV}$, and $2.2 \mathrm{kV}$.

Infectivity test. (i) In vitro. Infectivity of AmB-resistant promastigotes was determined classically with mouse peritoneal macrophages.

(ii) In vivo. Female $\mathrm{BALB} / \mathrm{c}$ mice, 18 to $20 \mathrm{~g}$ (Charles River, Cléon, France), were infected by the retroorbital sinus route with $10^{8}$ AmB-sensitive or AmBresistant promastigotes. Mice were sacrificed 4 weeks after infection. As $L$. donovani DD8 is poorly infective for animals, subcultures of liver and spleen were performed.

\section{RESULTS}

Phenotype of AmB-resistant promastigotes and in vivo infectivity. The growth rate of AmB-resistant promastigotes was slower (doubling time, $20 \mathrm{~h}$ ) than that of the wild-type (doubling time, $8 \mathrm{~h}$ ). The $50 \%$ inhibitory concentration of AmB was 20 times higher for resistant cells than for the wild-type. Resistant promastigotes responded to all drugs tested with a slightly decreased sensitivity to DFMO, sinefungin, and allopurinol and a significantly increased sensitivity to ketoconazole (Table 1). They were still able to infect macrophages in vitro but lost their virulence in vivo, since the subcultures of liver and spleen were negative for the AmB-resistant strain and positive for the AmB-sensitive strain. Furthermore, AmB resistance was conserved after culture in drug-free medium for more than 60 days.

Uptake of AmB in sensitive and resistant promastigotes. The intracellular AmB content in the L. donovani DD8 wild type increased with the incubation time, whereas that of the resistant $(\mathrm{R})$ cells increased slowly up to $8 \mathrm{~h}$ and decreased afterward (Fig. 1). As a function of extracellular AmB concentration, uptake was linear for wild-type promastigotes whereas for resistant promastigotes, the saturation was reached at 0.3 $\mu \mathrm{M}$ (Fig. 2).
TABLE 1. Effects of different drugs on in vitro growth of AmBsensitive and AmB-resistant L. donovani DD8 promastigotes after a $72-\mathrm{h}$ incubation period at $27^{\circ} \mathrm{C}$

\begin{tabular}{lcc}
\hline \multirow{1}{*}{ Drug } & \multicolumn{2}{c}{${\text { Mean } \mathrm{IC}_{50}{ }^{a} \pm \mathrm{SD}(\mu \mathrm{M})}$} \\
\cline { 2 - 3 } & $\begin{array}{c}\text { Amb-sensitive } \\
\text { cells }\end{array}$ & $\begin{array}{c}\text { Amb-resistant } \\
\text { cells }\end{array}$ \\
\hline Pentamidine & $2.7 \pm 0.8$ & $1.4 \pm 0.2$ \\
Allopurinol riboside & $1.1 \pm 0.2$ & $3.8 \pm 1.7$ \\
Taxotere & $27.7 \pm 10.6$ & $22.5 \pm 3.5$ \\
Paromomycin & $47.5 \pm 3.5$ & $41.0 \pm 11.7$ \\
DFMO & $225 \pm 35$ & $600 \pm 25$ \\
Sinefungin & $0.010 \pm 0.002$ & $0.58 \pm 0.11$ \\
AmB & $0.10 \pm 0.01$ & $1.89 \pm 0.12$ \\
Ketoconazole & $>1.9$ & $0.13 \pm 0.01$ \\
\hline
\end{tabular}

${ }^{a} \mathrm{IC}_{50}, 50 \%$ inhibitory concentration.

Efflux of AmB from sensitive and resistant promastigotes. No significant change was observed in the intracellular AmB content of wild-type promastigotes treated with $0.2 \mu \mathrm{M}$ of the drug for $2 \mathrm{~h}$ (Fig. 3). Under the same conditions, efflux of AmB from resistant promastigotes in drug-free culture medium increased as a function of incubation time, and no intracellular concentration was detected after 60 min (Fig. 3). However, when treated with 1 or $2 \mu \mathrm{M}$ AmB this efflux was slow (data not shown).

Lipids and membrane fluidity. The emission anisotropy value of AmB-resistant Leishmania promastigotes was five times lower than that of the wild-type promastigotes, indicating differences in the TMA-DPH environment within the plasma membranes of these cells. The membrane of AmB-resistant cells is therefore more fluid than that of AmB-sensitive cells (Table 2). This change in membrane fluidity is probably a consequence of the resistance-induced modification of membranous lipid metabolism. Nile red is a vital dye that emits a predominantly red fluorescence in polar hydrophobic domains (phospholipids) and a yellow fluorescence in neutral hydrophobic domains (14). Promastigotes in the logarithmic phase of growth, stained with Nile red and examined by light microscopy, showed that yellow fluorescence was emitted by intracytoplasmic lipidic droplets, whereas red fluorescence was localized in the membrane. No significant difference was observed between sensitive and resistant cells concerning membrane

Intracellular $[\mathrm{AmB}]$ (nmole/mg prot)

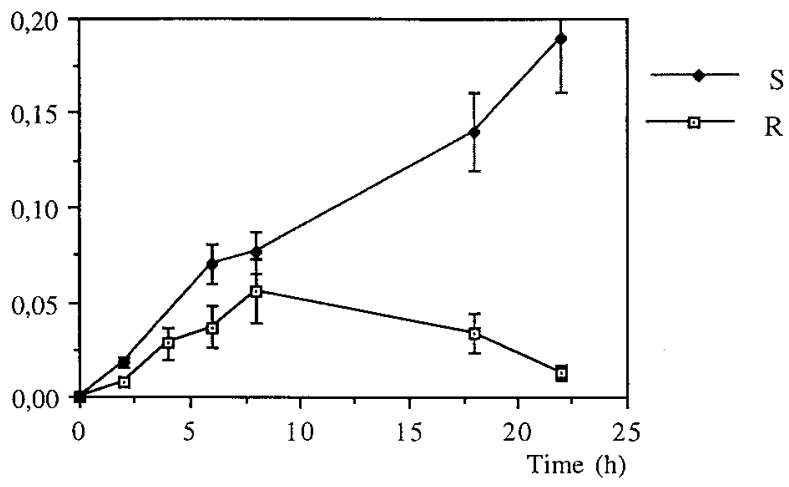

FIG. 1. Accumulation of AmB in L. donovani DD8-sensitive (S) and -resistant $(\mathrm{R})$ promastigotes after incubation with $0.2 \mu \mathrm{M}$ AmB. Values are means of three independent experiments, and standard deviations are represented by error bars. 


\section{A}

Intracellular $[\mathrm{AmB}]$ (nmole/mg prot)

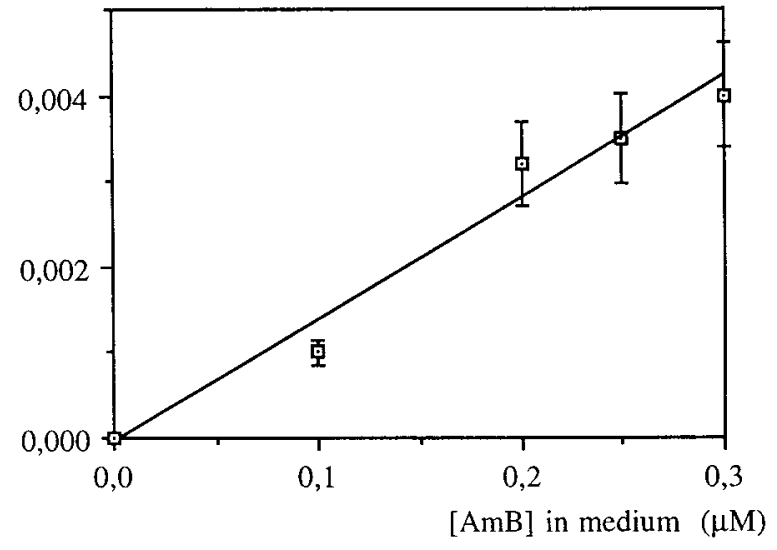

B

Intracellular $[\mathrm{AmB}]$ (nmole/mg prot)

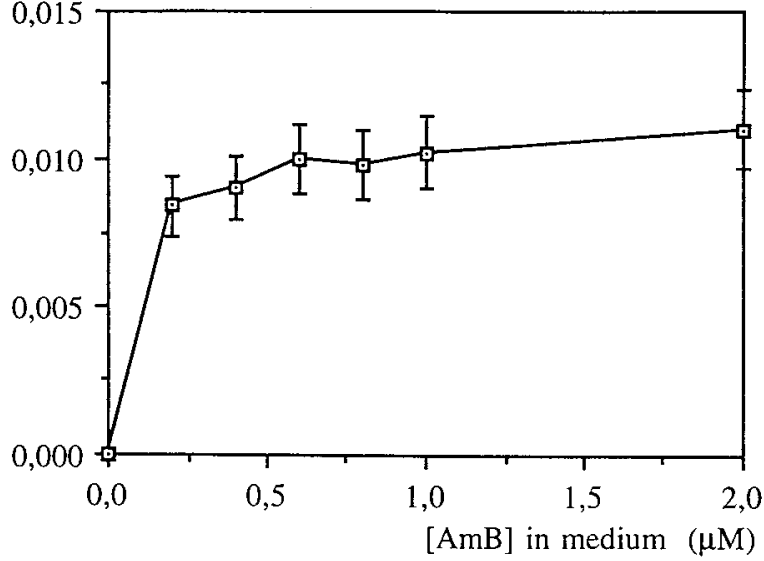

FIG. 2. Accumulation of AmB in L. donovani DD8-sensitive (A) and -resistant (R) promastigotes treated for $2 \mathrm{~h}$. Values are means of three independent experiments, and standard deviations are represented by error bars.

phospholipids, whereas the cytoplasm of AmB-resistant promastigotes contains less neutral lipids than that of the wild type.

Free sterol and fatty acid composition of $L$. donovani. GC/MS analysis showed that ergosterol (30\%) and its isomer, ergosta-5, 7, 24 (28)-trien-3ß-ol (32\%), represent $62 \%$ of total sterols in untreated wild-type promastigotes at logarithmicgrowth phase, whereas in AmB-resistant promastigotes, the major sterol is cholesta-5, 7, 24-trien-3 $\beta$-ol (58\%). These values increased in the stationary phase of culture (Table 3 ).

In ketoconazole-treated promastigotes, the sterol composition of AmB-resistant promastigotes, showed the following accumulation of methyl sterols: $14 \alpha$-methylcholesta- 8 , 24-dien$3 \beta$-ol, 33\%; $4 \alpha, 14 \alpha$-cholesta-8, 24-dien-3 $\beta$-ol, 5\%; $14 \alpha$-methylcholesta-5, 7, 24-trien-3 $\beta$-ol, 4\%; 4 $\alpha$, 4-dimethylcholesta- 8 , $24-$ dien-3 $\beta$-ol, $3 \%$ (Table 4 ). The corresponding accumulation for similarly treated wild-type promastigotes was as follows: $14 \alpha$-methylergosta-5, 7, 24 (28)-trien-3 $\beta$-ol, 35\%; $4 \alpha, 14 \alpha$-dimethylergosta-8, 24-dien-3 $\beta$-ol, 4\%; 14 $\alpha$-methylergosta- 8,24 (28)-dien-3 $\beta$-ol (1\%) (Table 4). Mass spectra for the TMS ether derivatives are given in Table 5.

Intracellular $[\mathrm{AmB}]$ (nmol/mg prot.)

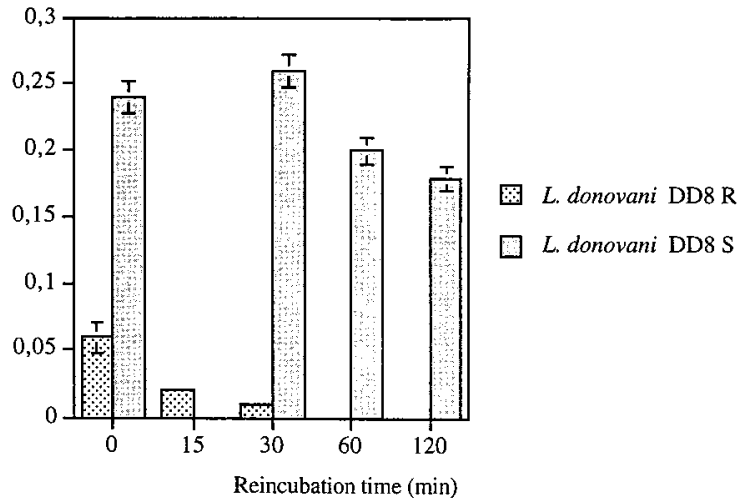

FIG. 3. Efflux of AmB from treated L. donovani DD8-sensitive (S) and -resistant (R) promastigotes for $2 \mathrm{~h}$ at $0.2 \mu \mathrm{M}$. Error bars indicate standard deviation.
Fatty acid composition was also different between the two cell types: stearic acid was the major fatty acid in resistant cells, whereas in wild-type cells, the unsaturated fatty acids were prevalent (Table 6).

\section{DISCUSSION}

Results presented in this paper provide some information on the mechanisms developed by $L$. donovani promastigotes to reduce the lethal effect of AmB. This resistance is stable even in the absence of drug pressure. The resistance observed to DFMO, sinefungin, and allopurinol riboside as well as the increased sensitivity to ketoconazole is probably related to altered membrane permeability as a consequence of the AmBinduced resistance.

Wild-type L. donovani promastigotes take up AmB for at least $24 \mathrm{~h}$. In contrast, AmB uptake in resistant promastigotes reached a maximum value after $8 \mathrm{~h}$ of incubation and then decreased. An obvious efflux of AmB was observed in resistant promastigotes.

Sterols of Leishmania species have been previously studied, and common characteristics in biosynthetic pathways have been found between Leishmania and fungi $(12,13)$.

Cholesterol is taken up from the culture medium but is not metabolized by promastigotes in either ketoconazole-treated or untreated cultures.

TABLE 2. Effects of AmB resistance on the fluorescence of exponential-phase $L$. donovani promastigotes labeled with Nile red and on the membrane fluidity of promastigotes as assessed by emission anisotropy of TMA-DPH

\begin{tabular}{lcccc}
\hline $\begin{array}{l}\text { Promastigote } \\
\text { characteristic }\end{array}$ & IRF $^{b}$ & IYF $^{c}$ & IYF/IRF & $r^{d}$ \\
\hline Amb sensitive & $50.2 \pm 1.2$ & $19.3 \pm 0.5$ & $92.8 \pm 0.6$ & $0.152 \pm 0.001$ \\
Amb resistant & $48.4 \pm 0.9$ & $15.6 \pm 0.1$ & $77.4 \pm 0.5$ & $0.031 \pm 0.001$ \\
\hline
\end{tabular}

${ }^{a}$ Cell staining and measurements were performed as described in Materials and Methods. Values are means \pm standard deviations for three experiments on untreated $L$. donovani DD8 AmB-sensitive and AmB-resistant promastigotes.

${ }^{b}$ IRF, intensity of red fluorescence.

${ }^{c}$ IYF, intensity of yellow fluorescence.

${ }^{d} r$, measure of fluorescence anisotropy of TMA-DPH. 
TABLE 3. Composition of free sterols in AmB-sensitive and -resistant L. donovani promastigotes

\begin{tabular}{|c|c|c|c|c|c|}
\hline \multirow{3}{*}{ Sterol $^{a}$} & \multirow{3}{*}{$\operatorname{Trr}^{b}$} & \multicolumn{4}{|c|}{$\%$ Total sterol } \\
\hline & & \multicolumn{2}{|c|}{ AmB-sensitive cells } & \multicolumn{2}{|c|}{ AmB-resistant cells } \\
\hline & & $\begin{array}{l}\text { Log } \\
\text { phase }\end{array}$ & $\begin{array}{l}\text { Stationary } \\
\text { phase }\end{array}$ & $\begin{array}{l}\text { Log } \\
\text { phase }\end{array}$ & $\begin{array}{l}\text { Stationary } \\
\text { phase }\end{array}$ \\
\hline Cholesterol (a) & 1 & 13 & 13 & 27 & 16 \\
\hline Ergosterol (b) & 1.34 & 30 & 31 & - & - \\
\hline Cholesta-5,7,24-trien-3 $\beta$-ol (c) & 1.15 & ${ }_{-c}^{c}$ & - & 58 & 66 \\
\hline Ergosta-5, 7, 24 (28)-trien-3 $\beta$-ol (d) & 1.53 & 32 & 36 & - & - \\
\hline 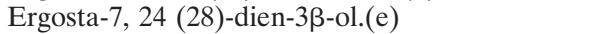 & 1.58 & - & $<1$ & - & - \\
\hline 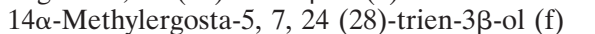 & 1.59 & 8 & - & - & - \\
\hline 4, $4 \alpha$-Dimethylergosta- $8,24(28)$-dien- $3 \beta$-ol (g) & 1.80 & - & 1 & - & - \\
\hline 24-Methylenedihydrolanosterol $(\mathrm{h})$ & 2.13 & - & $<1$ & - & - \\
\hline Other sterols (not identified) & & 17 & 18 & 15 & 18 \\
\hline
\end{tabular}

${ }^{a}$ Letters in parentheses are for ease of cross-reference among Tables 3 to 5 and Fig. 4.

${ }^{b}$ Trr, time of retention relative to cholesterol in GLC.

c 一, not detected.

In the L. donovani wild type, ergosterol and its isomer [ergosta-5, 7, 24 (28)-trien-3 $\beta$-ol] were present in the same proportion and were the major sterols, while in AmB-resistant promastigotes, the major sterol was cholesta-5, 7, 24-trien-3 ${ }^{-}$ol, which could produce ergosterol isomer by C-24 transmethylation. However, ergosterol was not detected. AmB-resistant Leishmania are therefore defective in C-24 transmethylation of C-27 sterols. This is consistent with the absence of C-28 sterols observed in resistant promastigotes (Fig. 4).

The analysis of sterol composition of wild-type promastigotes in stationary phase shows that the major sterols increased slightly and certain ergosterol precursors that were not observed at logarithmic phase accumulated. In AmB-resistant stationary-phase promastigotes, the major sterol, cholesta-5, 7, 24 -trien-3 $\beta$-ol, increased with the subsequent reduction of certain sterols.

These differences in sterol composition between logarithmic and stationary phases were expected and were also observed in Candida albicans (24).

L. donovani wild-type promastigotes were less sensitive to ketoconazole than were AmB-resistant cells. However, in both cell types the azole inhibited C-14 demethylation, as in fungi (34). In ketoconazole-treated AmB-resistant promastigotes, the methyl sterols which accumulated were the cholesta-5, 7, 24 -trien-3 $\beta$-ol precursors (i.e., $14 \alpha$-methylcholesta-8, 24-dien$3 \beta-o l)$. This confirms the above-mentioned sterol biosynthesis pathway where the end product is cholesta-5, 7, 24-trien-3 $\beta$-ol instead of ergosterol.

The interaction of $\mathrm{AmB}$ with ergosterol leads to the formation of transmembrane $\mathrm{AmB}$ channels which induce altered permeability to cations, water, and glucose and affect membrane-bound enzyme $(3,15)$.

Optimum interaction between a sterol molecule and AmB requires a $3 \beta-\mathrm{OH}$ group, a flat steroid nucleus, and a hydrophobic side chain at C-17 $(17,27)$. In addition, the length of the side chain allowing maximum interaction of AmB with cholesterol or its analogs containing dipalmitoylglycerophosphocholin liposomes must have more than five carbon atoms (25).

Optimum ergosterol-AmB interaction would probably require similar features in Leishmania or fungi. The minor modification of sterol structure, i.e., absence of methyl group at C-24 or double bond position in the side chain, would impair polyene-sterol interaction. The structural characteristics of the end product of sterol biosynthesis in AmB-resistant Leishmania fits this hypothesis. Indeed, the major sterol of AmB-resistant Leishmania differs from ergosterol of the wild type in

TABLE 4. Sterol composition of ketoconazole-treated AmB-sensitive and -resistant promastigotes ${ }^{a}$

\begin{tabular}{|c|c|c|c|c|c|}
\hline \multirow{3}{*}{ Sterol $^{b}$} & \multirow{3}{*}{$\operatorname{Trr}^{c}$} & \multicolumn{4}{|c|}{$\%$ Total sterol } \\
\hline & & \multicolumn{2}{|c|}{ AmB-sensitive cells } & \multicolumn{2}{|c|}{ AmB-resistant cells } \\
\hline & & Control & Exptl & Control & Exptl \\
\hline Cholesterol (a) & 1.00 & 13 & 18 & 27 & 14 \\
\hline 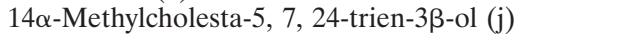 & 1.04 & $-^{d}$ & - & - & 4 \\
\hline 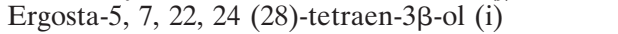 & 1.09 & - & 8 & - & - \\
\hline 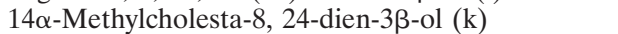 & 1.12 & - & 27 & - & 33 \\
\hline Cholesta-5, 7, 24-trien-3 $\beta$-ol (c) & 1.15 & - & - & 58 & 35 \\
\hline Ergosterol (b) & 1.20 & 30 & 6 & - & - \\
\hline 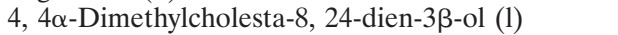 & 1.24 & - & - & - & 3 \\
\hline $4 \alpha, 14 \alpha$-Dimethylcholesta- 8,24 -dien-3 $\beta$-ol (m) & 1.26 & - & - & - & 5 \\
\hline 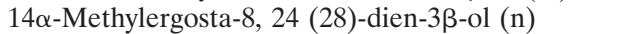 & 1.30 & - & 1 & - & - \\
\hline 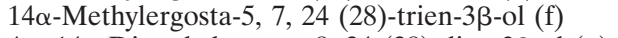 & 1.35 & 8 & 35 & - & - \\
\hline 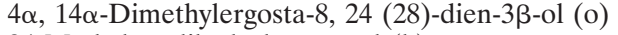 & 1.38 & - & 4 & - & - \\
\hline 24-Methylenedihydrolanosterol (h) & 1.54 & $<1$ & $<1$ & - & - \\
\hline
\end{tabular}

\footnotetext{
${ }^{a}$ Cells at logarithmic phase were treated for $24 \mathrm{~h}$ with $20 \mu \mathrm{M}$ (AmB sensitive) or $0.2 \mu \mathrm{M}$ (AmB resistant) ketoconazole.

${ }^{b}$ Letters in parentheses are for ease of cross-reference among Tables 3 to 5 and Fig. 4.

${ }^{c}$ Trr, retention time relative to cholesterol in GLC.

$d$ - not detected.
} 
TABLE 5. Mass spectrum data for TMS ether derivatives of sterols identified in $L$. donovani promastigotes ${ }^{a}$

\begin{tabular}{|c|c|c|c|c|c|c|c|c|c|c|c|c|c|c|}
\hline \multicolumn{15}{|c|}{ Fragment ions $(\mathrm{m} / \mathrm{z})$ in mass spectra of compound: } \\
\hline $\mathrm{a}$ & $\mathrm{b}$ & $\mathrm{c}$ & d & $\mathrm{e}$ & $\mathrm{f}$ & $\mathrm{g}$ & $\mathrm{h}$ & $\mathrm{i}$ & $\mathrm{j}$ & $\mathrm{k}$ & 1 & $\mathrm{~m}$ & $\mathrm{n}$ & o \\
\hline $458(13)$ & $468(20)$ & 454 (19) & $468(16)$ & $470(32)$ & $483(22)$ & $483(36)$ & $512(50)$ & $466(6)$ & $468(71)$ & $470(14)$ & $484(6)$ & $484(50)$ & 484 (11) & 498 (17) \\
\hline $444(3)$ & $454(5)$ & 439 (2) & $454(3)$ & $456(50)$ & $468(48)$ & 467 (44) & 497 (53) & $451(1)$ & $454(61)$ & $456(37)$ & $469(24)$ & $470(100)$ & $470(45)$ & $484(17)$ \\
\hline 369 (19) & 379 (21) & $364(21)$ & $378(20)$ & $414(3)$ & 451 (15) & $452(21)$ & 454 (73) & $393(1)$ & $364(100)$ & $366(10)$ & $454(100)$ & $415(10)$ & $213(7)$ & 394 (18) \\
\hline 354 (7) & $363(100)$ & 349 (100) & $363(100)$ & 387 (45) & 393 (11) & $429(10)$ & 441 (13) & 376 (11) & $338(5)$ & $342(1)$ & $426(64)$ & 380 (71) & $145(8)$ & 338 (1) \\
\hline $329(23)$ & 349 (15) & $324(67)$ & $337(68)$ & 380 (18) & $378(47)$ & $414(30)$ & $421(9)$ & $333(1)$ & $315(1)$ & $283(1)$ & $393(12)$ & $363(4)$ & 131 (11) & $309(2)$ \\
\hline $255(3)$ & $338(69)$ & 279 (4) & $294(10)$ & $372(17)$ & $363(100)$ & $393(21)$ & $394(20)$ & $281(5)$ & $294(2)$ & $213(2)$ & $364(58)$ & $323(2)$ & 119 (13) & $241(2)$ \\
\hline $207(3)$ & $223(9)$ & $251(30)$ & $279(8)$ & 363 (77) & $352(33)$ & $378(100)$ & $344(2)$ & $251(31)$ & 266 (1) & $159(6)$ & $308(2)$ & $304(30)$ & 105 (14) & 207 (4) \\
\hline $145(8)$ & $281(3)$ & $237(7)$ & $253(20)$ & 344 (100) & $338(51)$ & $364(24)$ & $298(3)$ & 209 (17) & $232(1)$ & $145(6)$ & $285(5)$ & $282(1)$ & $91(25)$ & $161(1)$ \\
\hline $129(38)$ & $253(37)$ & 225 (15) & $211(43)$ & 337 (43) & $279(5)$ & $351(40)$ & 257 (1) & $195(6)$ & 199 (2) & $131(8)$ & 254 (1) & $188(1)$ & $81(23)$ & $129(22)$ \\
\hline 119 (12) & $211(46)$ & 211 (43) & 185 (29) & $296(6)$ & 237 (3) & $325(8)$ & $205(100)$ & 147 (10) & 159 (2) & $129(7)$ & $179(2)$ & 107 (1) & $75(100)$ & 119 (11) \\
\hline $107(10)$ & $143(65)$ & 169 (39) & $168(36)$ & $255(13)$ & $195(7)$ & $294(7)$ & $172(1)$ & $129(7)$ & $140(3)$ & $119(7)$ & $147(3)$ & 89 (1) & & 109 (14) \\
\hline 95 (14) & $157(46)$ & $157(60)$ & $157(47)$ & $228(7)$ & $129(9)$ & $257(3)$ & 147 (19) & $93(3)$ & 114 (1) & 109 (11) & $106(1)$ & 69 (15) & & 105 (13) \\
\hline \multirow[t]{3}{*}{$73(72)$} & $143(65)$ & $143(90)$ & $143(76)$ & 213 (15) & 75 (10) & $199(5)$ & $133(21)$ & & & 105 (11) & & & & $91(16)$ \\
\hline & $131(57)$ & $131(68)$ & $133(60)$ & $145(13)$ & & $158(5)$ & $117(24)$ & $73(100)$ & & $95(14)$ & & & & $73(100)$ \\
\hline & $73(40)$ & $69(90)$ & $73(56)$ & $75(27)$ & & $75(6)$ & & & & $69(100)$ & & & & \\
\hline
\end{tabular}

${ }^{a}$ For pattern of fragmentation see references 10,12,19, and 22. Compounds a to o correspond to sterols listed in Tables 3 and 4 .

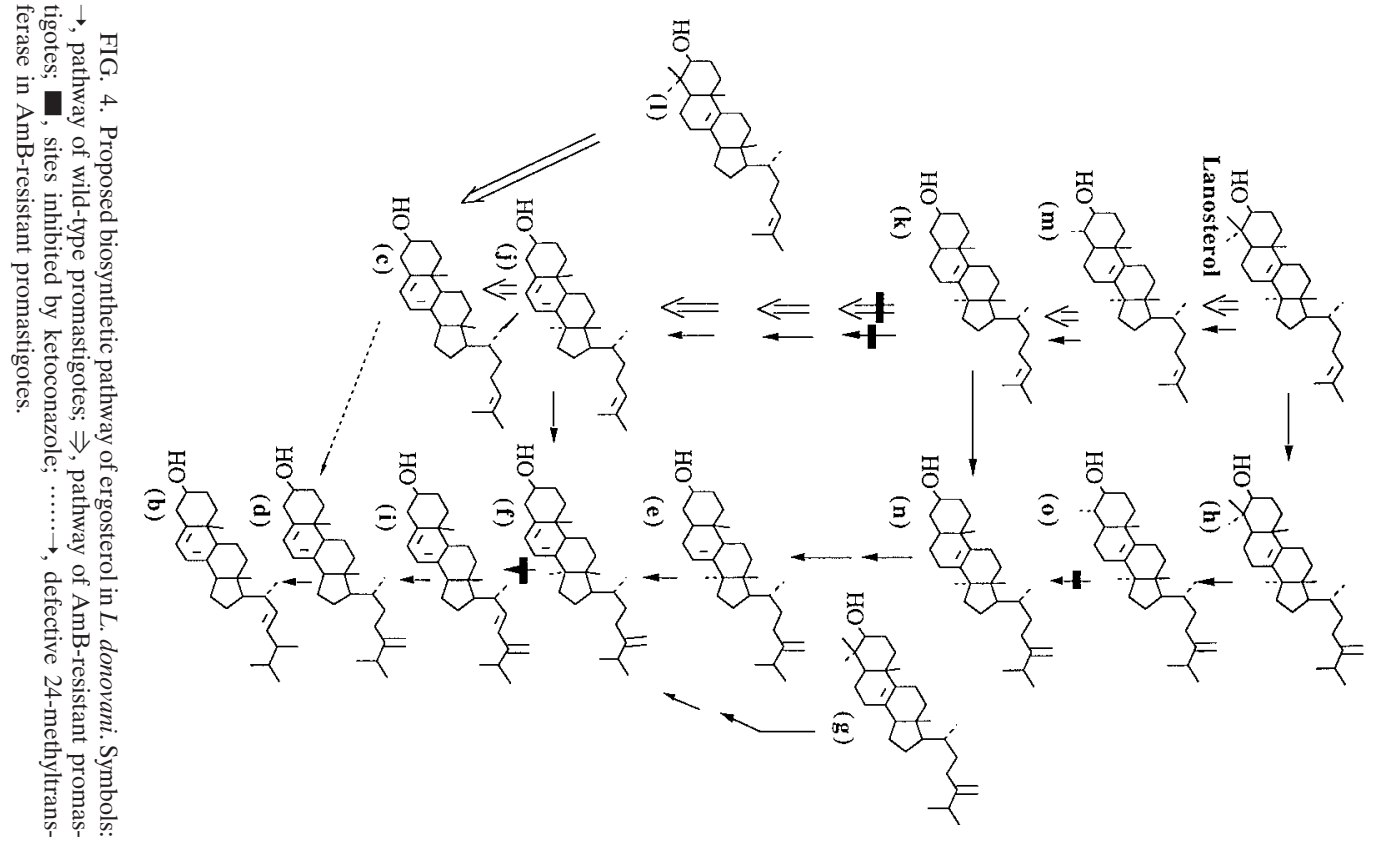

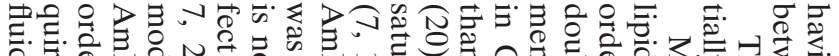

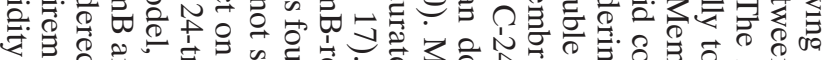
$\therefore 尹$

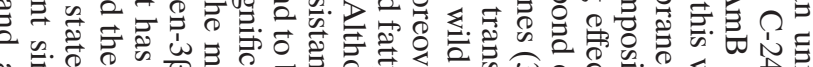

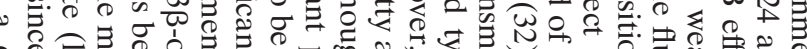

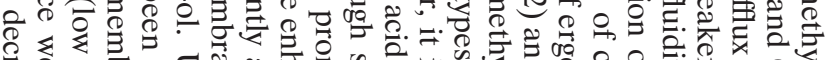
\&

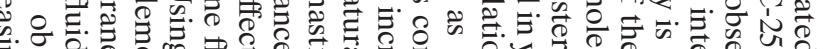

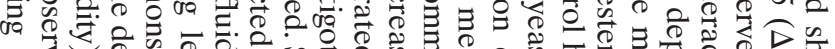

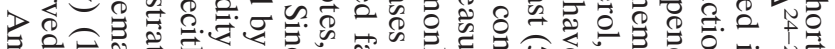
no

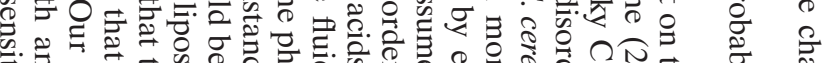

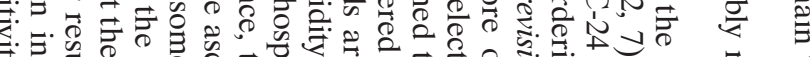

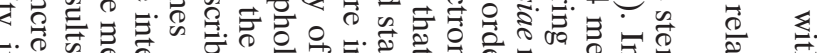

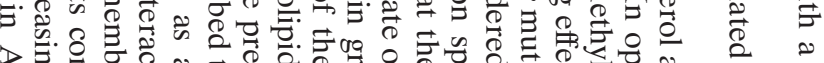

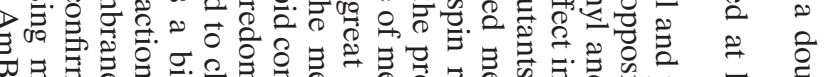

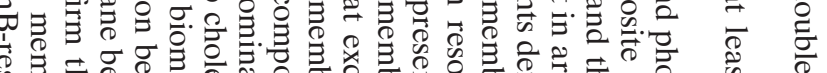
. 至. D.

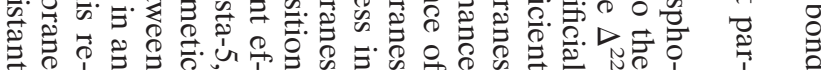


TABLE 6. Fatty acid composition of AmB-sensitive and -resistant $L$. donovani DD8 promastigotes

\begin{tabular}{|c|c|c|c|c|c|c|}
\hline \multirow{2}{*}{$\begin{array}{l}\text { Promastigote } \\
\text { characteristic }\end{array}$} & \multicolumn{5}{|c|}{$\%$ Total fatty acid of } & \multirow{2}{*}{$\begin{array}{l}\% \text { Saturated } \\
\text { fatty acid }\end{array}$} \\
\hline & $\begin{array}{l}\text { Myristate } \\
\text { (C-14:0) }\end{array}$ & $\begin{array}{l}\text { Palmitate } \\
\text { (C-16:0) }\end{array}$ & $\begin{array}{c}\text { Linoleate } \\
(\mathrm{C}-18: 2 \Delta 9,10-12,13)\end{array}$ & $\begin{array}{l}\text { Oleate }(\mathrm{C}- \\
18: 1 \Delta 9,10)\end{array}$ & $\begin{array}{l}\text { Stearate } \\
(\mathrm{C}-18: 0)\end{array}$ & \\
\hline AmB sensitive & 1 & 1 & 15 & 30 & 2 & 4 \\
\hline $\mathrm{AmB}$ resistant & $\ldots^{a}$ & 2 & - & 2 & 55 & 57 \\
\hline
\end{tabular}

${ }^{a}-$, not detected.

promastigotes. In the same study, it was also demonstrated that cholesterol decreased the order of dipalmitoyl-lecithin liposomes by reducing interactions between hydrocarbon chains of phospholipids (17). In AmB-resistant promastigotes, cholesta5, 7, 24-trien-3 $\beta$-ol could act similarly with fatty acids.

These observations suggest that the high membrane fluidity of AmB-resistant cells in comparison with the membrane fluidity of sensitive cells is due mainly to the presence of cholesta-5, 7, 24-trien-3 $\beta$-ol instead of ergosterol in the membranes.

In addition, the quantification of plasmic and intracytoplasmic lipids by fluorescence measurements indicates that their biosynthesis also undergoes some modifications in resistant cells.

Both AmB-sensitive and AmB-resistant promastigotes were infective for macrophages in vitro, whereas only the sensitive strain was infective in vivo. The greater microviscosity of parasite membranes is related to the higher exposure of membrane surface structures such as receptors, antigens, and enzymes (8). In AmB-resistant parasites, microviscosity as measured by fluorescence anisotropy was decreased. Therefore, membrane receptors were maybe not functional, explaining the lack of infectivity in vivo.

This study shows that resistance of $L$. donovani promastigotes to AmB involves the substitution of another sterol for ergosterol in the cell membrane, change in membrane fluidity, and a weak affinity of AmB for such modified membranes.

\section{ACKNOWLEDGMENT}

We thank S. L. Croft for critical reading of the manuscript.

\section{REFERENCES}

1. Berman, J. D., G. Ksionski, W. L. Chapman, V. B. Waits, and W. L. Hanson. 1992. Activity of amphotericin B cholesterol dispersion (Amphocil) in experimental visceral leishmaniasis. Antimicrob. Agents Chemother. 36:19781980.

2. Bloch, K. E. 1983. Sterol structure and membrane function. Crit. Rev. Biochem. 14:47-92.

3. Brajtburg, J., and J. Bolard. 1996. Carrier effects on biological activity of amphotericin B. Clin. Microbiol. Rev. 9:512-531.

4. Brassine, C., A. Laduron, A. Coune, J. P. Sculier, C. Hollaert, N. Collette, and F. Meunier. 1987. High-performance liquid chromatographic determination of amphotericin B in human serum. J. Chromatogr. 419:401-407.

5. Buttke, T. M., S. D. Jones, and K. Bloch. 1980. Effect of sterol side chains on growth and membrane fatty acid composition of Saccharomyces cerevisiae. J. Bacteriol. 144:124-130.

6. Davidson, R. N., S. L. Croft, A. Scott, M. Maini, A. H. Moody, and A. D. M. Bryceson. 1991. Liposomal amphotericin B in drug-resistant visceral leishmaniasis. Lancet 337:1061-1062.

7. Demel, R. A., and B. de Kruyff. 1976. The function of sterols in membranes. Biochim. Biophys. Acta 457:109-132.

8. Dutta, M., R. Bandyopadhyay, C. Ghosh, and M. K. Basu. 1990. Fluiditydependent $\mathrm{Mg}^{2+}$-ATPase activity in membranes from Leishmania promastigotes. Biochem. J. 265:923-926.

9. Fox, M. H., and T. M. Delohery. 1987. Membrane fluidity measured by fluorescence polarization using an EPICS V cell sorter. Cytometry 8:20-25.

10. Galli, G., and S. Maroni. 1967. Mass spectrometric investigations of some unsaturated sterols biosynthetically related to cholesterol. Steroids 10:189197.
11. Gantet, P., C. Hubac, and S. C. Brown. 1990. Flow cytometric fluorescence anisotropy of lipidic probes in epidermal and mesophyll protoplasts from water stressed Lupinius albus. L. Plant Physiol. 94:729-737.

12. Goad, L. J., G. G. Holtz, Jr., and D. H. Beach. 1984. Sterols of Leishmania species. Implications for biosynthesis. Mol. Biochem. Parasitol. 10:161-170.

13. Goad, L. J., G. G. Holtz, Jr., and D. H. Beach. 1985. Sterols of ketoconazoleinhibited Leishmania mexicana mexicana promastigotes. Mol. Biochem. Parasitol. 15:257-279.

14. Greespan, P., E. P. Mayer, and S. D. Fowler. 1985. Nile red: a selective fluorescent stain for intracellular lipid droplets. J. Cell Biol. 100:965-973.

15. Hartsel, S., and J. Bolard. 1996. Amphotericin B: new life for an old drug. Trends Pharmacol. Sci. 17:445-449.

16. Hebeka, E. K., and M. Solotorowsky. 1965. Development of resistance to polyene antibiotics in Candida albicans. J. Bacteriol. 89:1533-1539.

17. Hsu Chen, C. C., and D. S. Feingold. 1973. Polyene antibiotic action on lecithin liposomes: effect of cholesterol and fatty acyl chains. Biochem. Biophys. Res. Commun. 51:972-978.

18. Hülsewede, J. W., and H. Dermouni. 1994. Comparison of high-performance liquid chromatography and bioassay of amphotericin B in serum. Mycoses 37:17-21.

19. Knights, B. A. 1967. Identification of plant sterol using combined GLC/mass spectrometry. J. Gas Chromatogr. 5:273-282.

20. Lees, N. D., M. Bard, M. D. Kemple, R. A. Haak, and F. W. Kleinhans. 1979. ESR determination of membrane order parameter in yeast sterol mutants. Biochim. Biophys. Acta 553:469-475.

21. Maarouf, M., F. Lawrence, S. Brown, and M. Robert-Gero. 1997. Biochemical alterations in paromomycin-treated Leishmania donovani promastigotes. Parasitol. Res. 83:198-202.

22. Massey, I. J., and C. Djerassi. 1979. Structural and stereochemical applications of mass spectrometry in the marine sterol field. Synthesis and electron impact induced mass spectral fragmentation of $\Delta^{24}$ and $\Delta^{24(28)}-3 \beta$-hydroxy$\Delta^{5}$-sterols. J. Org. Chem. 44:2448-2456.

23. Mishra, M., V. Biswas, D. Jha, and A. Khan. 1992. Amphotericin versus pentamidine in antimony-unresponsive kala-azar. Lancet 340:1256-1257.

24. Mpona-Minga, M., A. Hakkou, J. Coulon, and R. Bonaly. 1988. Influence d'une dose subinhibitrice d'Amphotéricine B sur la composition en acides gras et stérols cellulaires de Candida albicans. Ann. Inst. Pasteur/Microbiol. 139:547-555.

25. Nakamura, T., M. Nishikawa, K. Inoue, S. Nojima, T. Akiyama, and U. Sankawa. 1980. Phosphatidylcholine liposomes containing cholesterol analogues with side chains of various lengths. Chem. Phys. Lipids 26:101-110.

26. Navin, T. R., B. A. Arana, F. E. Arana, J. D. Berman, and J. F. Chajon. 1992 Placebo controlled clinical trial of sodium stibogluconate (pentostam) versus ketoconazole for treating cutaneous leishmaniasis in Guatemala. J. Infect. Dis. 165:528-534.

27. Norman, A. W., R. A. Demel, B. DeKruijff, and L. L. M. Van Deenen. 1972. Studies on the biological properties of polyene antibiotics. Evidence for the direct interaction of Filipin with cholesterol. J. Biol. Chem. 247:1918-1929.

28. Pearson, R. D., and A. D. Sousa. 1996. Clinical spectrum of leshmaniasis. Clin. Infect. Dis. 22:1-11.

29. Phelouzat, M. A., F. Lawrence, and M. Robert-Gero. 1993. Characterization of sinefungin-resistant Leishmania donovani promastigotes. Parasitol. Res. 79:683-689.

30. Rodes, J. F., J. Berreur-Bonnenfant, A. Trémolières, and C. B. Spencer. 1995. Modulation of membrane fluidity and lipidic metabolism in transformed rat fibroblasts induced by the sesquiterpenic hormone farnesylacetone. Cytometry 19:217-225.

31. Russo, R., L. C. Nigro, S. Minniti, A. Montineri, L. Gradoni, L. Caldeira, and R. N. Davidson. 1996. Visceral leishmaniasis in HIV-infected patients: treatment with high dose liposomal Amphotericin B (AmBisome). J. Infect. 32:133-137.

32. Semer, R., and E. Gelerinter. 1979. A spin label study of the effects of sterols on egg lecithin bilayers. Chem. Phys. Lipids 23:201-211.

33. Thakur, C. P., M. Kumar, and A. Pandey. 1991. Comparison of regimens of treatment of antimony-resistant kala-azar patients: a randomized study. Am. J. Trop. Med. Hyg. 45:435-441.

34. Vanden Bossche, H., P. Marichal, and F. Odds. 1994. Molecular mechanisms of drug resistance in fungi. Trends Microbiol. 10:393-400. 